\title{
Les médecins communaux de Raguse (Dubrovnik) au Moyen Age
}

Mirko D. Grmek

\section{Summary}

Several medieval cities on the eastern coast of the Adriatic Sea renewed the Byzantine tradition of hiring a public physician, thus offering their citizens the service of qualified doctors. The case of Ragusa is typical. The archives of this city-state have been preserved since the XIIIth century; the names, origins and professional titles of public physicians are well known. The conditions of their employment reveal not only their duties and the salary but also many aspects of communal hygiene and medical ethics.

\section{Résumé}

Les conseils municipaux de plusieurs villes médiévales sur la côte orientale de l'Adriatique reprennent la tradition byzantine d'archiatre public et assurent aux citoyens les services de médecins qualifiés. A cet égard, le cas de Raguse est exemplaire. Les archives de cette ville-république ayant été conservées depuis la fin du XIII ${ }^{e}$ siècle, on connaît les noms, les origines et les qualifications des praticiens salariés par la commune. Les conditions de leur engagement renseignent non seulement sur leurs obligations et sur le montant de leur salaire mais aussi sur les débuts d'une sorte de sécurité sociale et sur certains aspects de l'hygiène communale et de l'éthique médicale.

Mirko D. Grmek, 10, rue de Savoie, F-75006 Paris 


\section{Introduction}

Avant d'entrer dans le vif du sujet, rappelons en quelques lignes l'histoire politique de la ville de Raguse (appelée Dubrovnik en croate) ${ }^{1}$. Ancienne agglomération romaine, slavisée au cours des siècles passées sous la domination byzantine et profitant des rivalités entre les puissances qui se disputent la côte dalmate, Raguse jouit d'emblée d'une grande autonomie municipale. Elle se constitue en petite république aristocratique et se soumet en 1205 à Venise pour se libérer en 1358 de la souveraineté de cette grande rivale. Du milieu du XIV ${ }^{\mathrm{e}}$ siècle jusqu'aux guerres napoléniennes, elle prospère comme une petite oligarchie ne reconnaissant que formellement l'autorité d'abord du roi de Hongrie et de Croatie, puis du sultan turc.

Tout comme les patriciens de Venise, la classe dirigeante de Raguse a très tôt compris l'importance politique et économique de la santé des citoyens. Pour ces administrateurs pragmatiques il était évident que l'Etat devait s'occuper de la santé au même titre qu'il lui incombe de maintenir l'ordre public, de diriger les relations internationales et de veiller sur les intérêts commerciaux $^{2}$. L'exemple de la politique sanitaire de Venise et de Raguse a été suivi par plusieurs villes de Dalmatie, jalouses de leurs anciennes prérogatives d'autonomie municipale et soucieuses de ne pas être dépassées par les réalisations des voisins. Dans cette saine émulation excellaient non seulement Zadar et Split, mais aussi Kotor, Trogir, Sibenik, Korcula, Hvar et Rab33.

1 Les deux noms de cette ville de la côte dalmate remontent aux temps de sa fondation. Au $\mathrm{VII}^{\mathrm{e}}$ siècle, après les pillages de la ville d'Epidaurum (aujourd'hui Cavtat, c'est-à-dire Civitas vetus) par des Slaves, ses habitants romans se sont refugiés dans un lieu fortifié voisin, bâti sur des rochers et appelé Rausium ou Ragusium. Sans doute le nom de Dubrovnik (étymologiquement le lieu dans la forêt) désignait initialement le village slave situé sur la côte face aux fortifications. L'agglomération urbaine Raguse-Dubrovnik résulte de l'unification de ces deux localités adjacentes et de la slavisation de leurs habitants pendant la période historiquement opaque qui va du $\mathrm{VII}^{\mathrm{e}}$ au XII $\mathrm{XI}^{\mathrm{e}}$ siècle.

2 Pour l'histoire de la médecine et des conditions sanitaires à Raguse au Moyen Age, voir en particulier R. Jeremić et J. Tadić J., Prilozi za istoriju zdravstvene kulture starog Dubrovnika, 3 vols., Beograd, 1938-1940 (= J.-T.); M. D. Grmek, «Medizin und Pharmazie in der ehemaligen Republik Dubrovnik», Hauptversammlung der Internationalen Gesellschaft für Geschichte der Pharmazie (Dubrovnik, 1959), Stuttgart,1960,51-59; M. D. Grmek, «Povijest medicine u Dubrovniku», Medicinska Enciklopedija, vol. II, Zagreb, 1967, 235-237; L. Glesinger, «La médecine dans l'ancien Dubrovnik», in Veteris Ragusae Medicina et Pharmacia, Zagreb, s. a., 63-77; V. Bazala, Pregled povijesti zdravstvene kulture Dubrovačke Republike, Zagreb, 1972; S. M. Stuart, «A Communal Program of Medical Care; Medieval Ragusa-Dubrovnik», Journal of the History of Medicine, 28, 1973, 126-142; M. D. Grmek, «Medicina i ljekarništvo u negdašnjoj Dubrovačkoj Republici», Revue Dubrovnik, 3, 1992, n 2-3 325-331; J. Bačic, «Medicine in Dubrovnik Until the 1667 Earthquake», Croatian Medical Journal, 35, 1994, 113-120.

3 Pour l'histoire de la médecine dans le cadre municipal de ces villes, voir notamment V. Brunelli, Storia della città di Zara, Venezia, 1913; G. Novak, «Liječnici, brijači i ljekarnici autonomnog Splita», Liječnički Vjesnik, 53, 1931, 401-403; M. D. Grmek, «Povijest zdravstva na 
Les médecins qui exerçaient dans ces villes entre le XIII' et le XVI ${ }^{e}$ siècle n'avaient pas tous le même niveau d'éducation professionnelle, ni le même statut social. La plupart des praticiens de l'art de guérir étaient sans qualifications particulières. C'étaient d'abord les guérisseurs et les «femmes habiles» (vještice, terme qui finira par signifier «sorcière»); puis de nombreux prêtres et moines pratiquant la médecine sans formation professionnelle; enfin les barbiers et les pharmaciens. Dès le XIII ${ }^{\mathrm{e}}$ siècle, les statuts des villes dalmates condamnent la sorcellerie, le charlatanisme et l'exercice lucratif de la médecine populaire, ce qui prouve l'existence, à ce moment déjà et au moins dans les zones urbanisées, de médecins formellement qualifiés. En Croatie médiévale, à l'instar des autres pays européens, ces médecins n'avaient pas tous le même rang social. On distinguait notamment les physiciens des chirurgiens. Les premiers étaient peu nombreux; leur aide était relativement chère, accessible seulement à une partie restreinte de la population générale. Ils étaient érudits mais leurs interventions médicales se limitaient aux conseils. Les seconds, socialement inférieurs, soignaient en utilisant non seulement leur savoir mais aussi leurs mains. La limite entre les chirurgiens d'un côté et les barbiers et les rebouteux de l'autre n'était pas toujours nette.

$\mathrm{C}^{\prime}$ est par le recours à l'ancienne tradition byzantine des archiatres communaux, c'est-à-dire des médecins payés pour demeurer en un lieu déterminé, que les conseils municipaux assurent, au moins dès la fin du XIII ${ }^{\circ}$ siècle, la présence constante de physiciens et de chirurgiens qualifiés dans les principales villes de Dalmatie. A cet égard, le cas de Raguse est emblématique.

\section{Un témoignage littéraire sur les médecins salariés}

En 1440, Philippe de Diversis, professeur (rector scholarum) salarié au service de la République, rédige une description de cet Etat, de son organisation et des mœurs de ses habitants - description dans laquelle tout un chapitre est consacré aux médecins salariés. Voici notre traduction de ce texte

Rabu», Medicinar (Zagreb), 3, 1949, 291-302; M. D. Grmek, «Pregled povijesti zdravstvenih prilika u Zadru», Liječnički Vjesnik, 72, 1950, 178-184; R. Ferri, «Zdravstveni propisi dalmatinskih statuta iz Srednjeg vijeka», in M. D. Grmek \& S. Dujmušic (réd.), Iz hrvatske medicinske proslosti, Zagreb, 1954, 138-146; M. Škarica, «Zadarski liječnici», Radovi Instituta JA u Zadru, 2, 1955, 141-167; R. Kovijanić et M. Stjepčević, Kulturni život staroga Kotora, II, Cetinje, 1957; D. Berić, «Liječnici, apotekari i brijači autonomnog Splita», Anali Historijskog Instituta u Dubrovniku, 6-7, 1959, 283-291; A. Kapor, Zdravstvene prilike u Korčuli u srednjem vijeku, Korčula, 1971; M. D. Grmek, «Zdravstvene prilike i medicina u Šibeniku do početka XX stoljea», Spomen-zbornik o 900 obljetnici Šibenika, Šibenik, 1976, 521-539; R. Jelić, Zdravstvo u Zadru i njegovom području, Zadar, 1978. 
latin, resté inédit jusqu'à la fin du XIX ${ }^{\mathrm{e}}$ siècle et ignoré des historiens de la médecine ${ }^{4}$ :

De l'habitude louable d'avoir, d'honorer et de rétribuer quatre médecins. - Tous les auteurs catholiques et philosophes païens s'accordent pour dire qu'il faut honorer les médecins qui soignent les maladies du corps et le plus souvent, par la grâce de Dieu, rendent la santé. Le miséricordieux Créateur du monde, ayant eu pitié de la faiblesse humaine, a doué les gens sages des lumières de la raison pour leur faire connaître la composition du corps, les propriétés et les pouvoirs des herbes, des semences et d'autres substances pour qu'ils puissent choisir ce qui est utile pour la santé et écarter ce qui est nuisible. Puisque existent donc des médecins utiles et même nécessaires à toute ville illustre, il faut louer l'habitude du Grand Conseil ragusain d'avoir à son service, aux frais de la municipalité, quatre médecins, à savoir deux physiciens, l'un plus érudit que l'autre, et deux chirurgiens. Chacun d'eux est obligé par la loi, par les accords et par contrat, de soigner, sans honoraires privés, consciencieusement, attentivement et gratuitement tous les Ragusains, dans la ville ou en dehors d'elle, nobles et fonctionnaires, pauvres et riches, ainsi que leurs familles. Il doit faire de son mieux pour rendre la santé aux malades dans tous les cas où c'est possible. Car juste est la sage pensée du philosophe: De même qu'aucun orateur ne convaincra toujours, aucun médecin ne guérira toujours. Toutefois, s'il ne néglige rien de ce qu'il doit faire, on dira qu'il connaît bien son métier. Il ne me revient pas, il n'est pas nécessaire et d'ailleurs je n'y tiens pas, de révéler quel salaire chacun d'eux perçoit de la Commune. Je dirai seulement que leurs salaires atteignent un montant digne des médecins les plus illustres. En outre, ils reçoivent à tout moment quantité de cadeaux de la part de leurs patients. Et quand ils visitent des étrangers malades, ces derniers les payent. Tous les citoyens de Raguse, quel que soit leur statut social, respectent et honorent les médecins, certains poussés par la souffrance, d'autres par la reconnaissance de leurs qualités ou le désir de contribuer à leur réputation. Car aucune renommée n'est plus méritée, plus durable ni plus justifiée que celle obtenue, accrue et répandue par l'opinion des gens sages et vertueux. Si tout cela mérite un éloge particulier, il ne faut pas oublier de mentionner également un autre fait louable dans l'attitude des Ragusains envers leurs médecins. Ils avaient l'habitude de ne pas licencier leurs médecins salariés en les laissant démunis. Quant au physicien ou au chirurgien les ayant servis et ne pouvant plus exercer du fait de son âge ou d'une maladie, ils considèrent qu'eu égard aux services rendus pendant sa jeunesse, il serait mal de le licencier. A celui-ci, ils accordent une pension municipale lui permettant de subvenir à ses propres besoins et à ceux de sa famille jusqu'à la fin de sa vie, libre de toute obligation de soigner, à moins qu'il y trouve un plaisir personnel. A ce que j'ai entendu, cette pension a été donnée à plusieurs personnes ; moi-même, j'ai vu un tel cas. Parmi les premiers, je cite l'exemple d'un chirurgien, maître Jean de Aldoardis de Pavie qui était, dit-on, le meilleur médecin dans sa branche. Devenu vieux, il a eu une retraite à vie. De mon temps, on a accordé la même chose à un autre. Son nom est Jean de Teolo, originaire de Padoue, lui aussi chirurgien. Vaincu moins par la vieillesse que par la maladie l'obligeant à rester la plupart du temps au lit ou au moins chez lui, il a pris conscience de son incapacité et a demandé au Sénat d'être relevé de ses fonctions. Par la décision du Grand Conseil, suite à la proposition et à la recommandation du Petit Conseil, on lui a accordé une pension, à vie, de cinquante ducats d'or annuels. J'ai entendu dire qu'il y aurait des cas où l'on agit de même avec certains scribes et notaires, mais je n'ai jamais ni vu ni entendu qu'on aurait agi ainsi à l'égard d'autres salariés. Ainsi tous les médecins et secrétaires du Conseil peuvent garder bon espoir. Les vertus et les mérites des autres, à ce qu'il semble, ne sont pas reconnus de la même manière.

De Diversis termine par un plaidoyer pro domo car, maître d'école, il aurait voulu que son métier soit traité comme celui des médecins. Le chapitre suivant s'intitule d'ailleurs: Des maîtres salariés qui enseignent la jeunesse. No-

4 Situs aedificiorum, politiae et laudabilium consuetudinum inclytae civitatis Ragusii,lib. III,cap. 7; publié par V. Brunelli, dans Programma dell'Imp. Reale Ginnasio Superiore di Zara,Zadar, 1882 
tons que, à plusieurs reprises, aussi bien à Raguse que dans les autres villes de Dalmatie, notamment à Split, les postes d'instituteurs étaient confiés aux médecins, soit parallèlement à leurs fonctions médicales, soit en alternance avec elles.

Les sources d'archives confirment entièrement le témoignage de De Diversis et donnent une image étonnament riche et avancée de la santé publique dans la vieille Raguse. Ainsi, il résulte des documents conservés dans les Archives d'État de Dubrovnik que Jean de Aldoardis de Pavie, médecin chirurgien (medicus cerusicus), est embauché le 24 juillet 1376 comme employé communal avec un salaire annuel de 200 ducats d'or. Lorsque, après presque quarante ans de fidèles service, maître Jean déclare forfait par faiblesse physique, le Grand Conseil de Raguse lui attribue, le 26 février 1415, une pension de 200 perpères $^{5}$, payable annuellement jusqu'à la fin de ses jours ${ }^{6}$. Cette décision a été prise en soulignant bien qu'il s'agit non pas d'un droit ou de l'exécution d'une clause contractuelle mais d'une faveur accordée pro remuneratione benemeritorum suorum et pro honore rei publicae ${ }^{7}$.

Quant à Jean de Teolo, chirurgien originaire de Padoue, il est venu à Raguse en 1423 et a passé douze ans au service de la République, avant de demander son licenciement pour cause de maladie. Le 9 avril 1435, maître Jean de Padoue a obtenu une pension annuelle à vie, en promettant toutefois de ne pas quitter la ville et de continuer à soigner les citoyens selon ses forces et son bon plaisir. Son salaire se montait à 220 ducats d'or, mais sa pension n'atteignait que 50. Décédé en avril 1438, il a joui de cette pension pendant trois ans ${ }^{8}$.

\section{Les plus anciennes informations sur les médecins communaux de Raguse}

Il nous faut revenir en arrière. A Raguse, comme dans les autres anciennes municipalités dalmates, les premières informations sur les médecins salariés datent de la fin du XIII ${ }^{\mathrm{e}}$ siècle. Leur apparition coïncide en fait avec le début de la documentation susceptible de signaler leur existence. Les plus anciens

5 Notons que les perpères (ou hyperpères), anciennes pièces d'or byzantines, étaient à cette époque une monnaie fictive dont se servaient les Ragusains pour standardiser leur comptabilité. Une perpère équivalait à environ un tiers du ducat d'or. La pension de Jean de Pavie correspondait donc à environ un tiers de son salaire de chirurgien en activité.

6 Archives d'Etat de Dubrovnik ( = AED), Diversa Cancellariae, 24, 167v. - Voir J.-T., II 15-16, et III 134.

7 AED, Reformationes, Consilium Maius, 31, 161 et 174; Consilium Rogatorum, 30, 76. - J. T., ibid.

8 AED, Consilium Maius, 3, 18 et 211v, 5, 30v-31; Diversa Cancellariae, 46, 132v; Diversa Notariae, 22, 10v. - J.-T., II 30 et III 138. 
actes des notaires et les premiers comptes rendus des délibérations municipales mentionnent déjà les physiciens et les chirurgiens salariés. Il s'agit bien entendu des premiers documents de ce genre conservés et non pas premiers au sens absolu. L'institution des médecins salariés précède donc notre documentation historique; ses débuts se perdent dans les temps opaques.

Les séries continues des actes conservés aux archives historiques de Dubrovnik remontent à 1278. Le premier médecin dont le nom apparaît dans ces documents est maître Jean de Trévise (magister Johannes de Trevisio medicus), mentionné dès 1279. Italien d'origine, il avait épousé une Croate du nom de Desislava9.

A la fin du XIII ${ }^{\mathrm{e}}$ siècle vivait à Raguse un médecin dont le nom slave, Prvoslav (Pervosclavus Rubeus medicus), indique clairement l'origine locale. Sa situation sociale était probablement modeste, car un document de 1281 parle de sa «cabane» (capanna). Rien n'est connu de son activité médicale. Sans doute était-il un simple empirique. Sa femme, Prvica, figure en 1298 comme veuve (Perviça uxor quondam Pervosclavi medici) ${ }^{10}$.

Pour les médecins postérieurs à 1300 , nous ne prendrons en considération que ceux dont l'activité salariée est expressément rappelée dans les actes conservés. Le premier de cette liste est un chirurgien, medicus plagarum. On connaît son nom, Marc, mais pas son origine. Marc était un prénom très commun non seulement à Venise mais aussi dans toute la Dalmatie.

Voici le texte précisant les conditions de son embauche:

1301. Le 18 septembre courant. Marc, médecin des plaies (magister Marcus, medicus plagarum) est confirmé, par ordre de Monsieur le Duc et de son Petit Conseil, après l'autorisation du Grand Conseil, comme salarié de la commune de Raguse (firmatus fuit ad salarium comunis Ragusii) pour deux ans et pour la somme annuelle de 8 livres vénitiennes, et un logement comme il en jouit déjà maintenant, et ce à dater du 18 de septembre courant ${ }^{11}$.

Il est probable que, avant cette date, Marc était déjà au service de la ville. On ne dispose que d'une documentation très incomplète sur les décisions antérieures à 1300. A l'expiration des deux années prévues, Marc est licencié. Le Grand Conseil ordonne, en septembre 1303, à ses émissaires de s'enquérir à Venise d'un nouveau chirurgien.

En janvier 1304, on confie la charge de chirurgien communal à maître Etienne (magister Stephanus medicus plagarum $)^{12}$, peut-être un Ragusain ou du

9 AED, Reformationes 54, 217. - Čremošnik G., Kancelariski i notarski spisi, Beograd, 1932 (= Čremošnik, 1932), no 323; G. Čremošnik, Spisi dubrovačke kancelarije, Zagreb, 1951 (= Čremošnik, 1951), 14 et 48; J.-T. II 7 et III 126.

10 AED, Debita Notariae 1, 64. - Čremošnik, 1951, 64; J.-T., II 7 et III 126.

11 Monumenta Ragusina ( = Mon. Rag.), V 6 et 11. - J.-T., II 7 et III 126.

12 Mon. Rag. II 304. - J.T., II 8 et III 127 (par une mauvaise lecture des actes, on a fait par erreur d'une seule personne deux chirurgiens différents, Stephanus et Estesanus). 
moins un homme bien intégré dans cette ville, comme le prouve le nom typiquement ragusain de son fils Blaise (Vlaho), mentionné comme orfèvre en 1325. En août 1305, le Grand Conseil embauche à nouveau Marc, mais son contrat est cassé avant l'échéance prévue. Les démêlés de Marc avec la justice prouvent qu'il vit encore à Raguse en 1312, désormais sans emploi fixe ${ }^{13}$. Le chirurgien Etienne y exerce au moins jusqu'en 1313, lui aussi sans charge communale.

Maître Etienne a promis par un contrat passé devant notaire le 6 novembre 1305 de soigner Dragosh de Bosnie, blessé au genou par une flèche. Le chirurgien retirera le fer resté dans la blessure et guérira la fistule. S'il réussit, il recevra 30 perpères; s'il échoue, il ne recevra rien, ni pour ses efforts, ni pour les médicaments utilisés. Toutefois, le malade lui donne immédiatement en gage plusieurs objets en or et en argent ${ }^{14}$. Par un autre contrat, signé le 30 août 1313, le même chirurgien promet de guérir, dans un délai de quatre mois, le citoyen ragusain Clément Lukarevich de ses plaies et de ses fistules à la jambe. Dès que l'os sera sans pus, Etienne recevra 5 perpères et après la guérison complète du malade 5 autres perpères. Le chirurgien promet de ne pas se servir du couteau, ni du cautère, tandis que le patient s'engage à suivre fidèlement le traitement prescrit ${ }^{15}$.

Le chirurgien Marc, lui aussi, a promis par un contrat enregistré au tribunal le 3 juin 1306 de guérir le fils de Milosh Ljubenov de Korcula qui souffre de plusieurs fistules au-dessous du genou ${ }^{16}$. S'il réussit, il recevra 15 perpères; s'il ne réussit pas dans le courant d'une année, il n'aura rien; si la maladie récidive, il rendra l'argent reçu ${ }^{17}$. La suite des actes d'archives montre que Marc a effectivement guéri ce garçon, atteint sans doute d'une ostéomyélite et venu d'une île dalmate à Raguse dans l'espoir d'y recevoir la cure salvatrice.

En octobre 1301, le Grand Conseil de Raguse accorde au maître Mertacha lui aussi medicus plagarum, la somme de 30 perpères pour un an de service, suivant l'habitude. Ce chirurgien doit jurer qu'il soignera bien, selon les lois de l'art et au mieux de son savoir tous ceux qui s'adresseront à lui. La charge municipale de ce médecin est prolongée, d'année en année, cum conditionibus consuetis, jusqu'au novembre $1313^{18}$.

La ville de Raguse avait donc à son service, pendant les premières années

13 Mon. Rag. V, 66 et 104.

14 AED, Diversa Cancellariae 4, 79. - Čremošnik, 1931,44; M. D. Grmek, «Srednjovjekovni ugovori o liječenju s hrvatskog područja», in S. Dujmušić et M. D. Grmek (réd.), Iz hrvatske medicinske prošlosti, Zagreb, 1954, p. 155.

15 Čremošnik, 1931, 43.

16 AED, Diversa Cancellariae 4, 101.

17 AED, Diversa Cancellariae 4, 101' . - Čremošnik, 1931, 44; Grmek, 1954, 155.

18 Mon. Rag. V 11. - J.-T., II 7-8, III 126-127. 
du XIV ${ }^{e}$ siècle, deux chirurgiens. Un acte particulièrement instructif prouve qu'elle s'assurait en même temps le service d'un médecin-physicien compétent:

1302. Dimanche, 2 septembre. Maître Richard médecin physicien (magister Ricardus medicus physicus), est confirmé, à la séance du Grand Conseil, comme salarié de la commune de Raguse pour deux ans et pour la somme annuelle de 140 perpères et un logement convenable à la charge de la commune, et ce à la condition que, pour ses cures, il ne reçoive de paiement d'aucun Ragusain et qu'il conseille et traite bien et selon l'art tous ceux qui l'appelleront, aussi bien les riches que les pauvres; et il ne doit s'associer avec aucun apothicaire pour vendre dans sa boutique des médicaments ou autres objets. Pour son travail, il peut recevoir des étrangers des sommes convenues avec eux ${ }^{19}$.

Ce Richard venait de Salerne, entouré du prestige que conférait encore cette célèbre école de médecine. Il reste au service de Raguse au moins jusqu'en 1314. Pendant l'été 1304, le gouvernement lui permet de se rendre dans la région de la Neretva pour soigner pendant quinze jours le potentat croate Constantin Nelipich. Au cas où il prolongerait son absence de Raguse, une somme en rapport avec la durée de celle-ci sera déduite de son salaire ${ }^{20}$.

Un seul médecin-physicien ne suffisant pas à Raguse alors en pleine expansion économique, le Grand Conseil embauche en 1304, à côté de Richard de Salerne, maître Nicolas, originaire des Marches d'Ancône. Son salaire n'atteint que la moitié de celui de Richard, mais il garde le droit de se faire payer par les malades qui le consultent, à condition toutefois que ses honoraires ne dépassent pas 2 perpères par traitement ${ }^{21}$.

Maître Nicolas ne reste que peu de temps à Raguse. La commune entre donc en pourparlers avec maître Pierre Maranzio, physicien de Salerne. Pour attirer cet illustre praticien ou l'un de ses collègues, le Grand Conseil est prêt, d'après le vote du 25 mai 1305, à offrir un salaire annuel allant jusqu'à 500 perpères (frais de logement compris), avec la possibilité, pour le médecin, de se faire en outre payer par les malades (les honoraires ne devant pas dépasser 2 perpères pour les Ragusains mais restant libres pour les étrangers). La dernière clause des propositions ragusaines est révélatrice: le médecin doit s'engager «à visiter personnellement les malades, notamment les nobles de la ville» ${ }^{22}$.

La documentation des Archives d'Etat de Raguse n'est pas complète pour cette période et pendant plusieurs années, jusqu'en 1322, manquent des informations sur les physiciens communaux. En revanche, il est assez souvent

19 AED, Reformationes, 2, 59. - M. D. Grmek, «Influence de l'Ecole de Salerne sur le développement de la médecine en Croatie», Scalpel, 106, 1953, 33-37, et «Salerno i Hrvatska», Liječnički Vjesnik, 75, 1953, 259-265.

20 Mon. Rag. V 76.

21 Mon. Rag. II 307 et V 78. - J.-T., II 8 et III 128.

22 Mon. Rag. V 88. - J.-T., II 8-9 et III 128. 
question des chirurgiens. On les désigne par des termes cirologus, cirolocus, ciroicus, cerusicus, cyrugicus, medicus cyrugie, medicus in arte cirologie ou simplement medicus. En 1313, la dernière année du service de maître Mertacha, le Petit Conseil embauche provisoirement, le 25 avril, pour un seul mois, maître Barthélemy. Il reçoit un salaire apparemment très élevé, à savoir 20 perpères pour 30 jours, mais il doit «soigner avec application tous les habitants de la ville de Raguse et de son district, notamment tous les citoyens, Monsieur le Duc et sa maisonnée, Monsieur l'Archevêque et sa maisonnée, tous les religieux et toutes les religieuses et tous les salariés municipaux et leurs familles, sans aucune récompense particulière». En outre, maître Barthélemy doit fournir gratuitement à toutes ces personnes des emplâtres, des onguents, des eaux médicinales et tous les médicaments afférents à l'art chirurgical ${ }^{23}$. Satisfaits de ses prestations, les Ragusains prolongent le contrat de ce chirurgien avec un salaire annuel.

Ils essayent à plusieurs reprises de convaincre maître Albert, chirurgien salarié à Venise, de changer d'employeur. Fort de l'offre ragusaine, Albert obtient habilement que les Vénitiens lui augmentent son salaire et envoie à Raguse, à sa place, son frère Bonaventure. Le Grand Conseil prend ce dernier à son service comme chirurgien communal avec un salaire annuel de 12 livres, augmenté de 20 sous vénitiens pour frais de logement, mais lui fait jurer sur la Bible qu'il accepte de soigner gratuitement «tous les habitants de la ville et du district de Raguse, assurant à ses propres frais les onguents, les emplâtres, les eaux médicinales et tous ce qui est nécessaire pour l'exercice de l'art chirurgical» ${ }^{24}$.

Pendant les quarante dernières années de la domination vénitienne, c'està-dire entre 1319 et 1358 , ont travaillé à Raguse comme chirurgiens salariés les maitres suivants (la date entre parenthèses indique la première mention de leur service): Jacques de Forli (1319), Jean des Pouilles (1320), Thomas (1323), Dominique (1323), Bénoit de Fano (1329), Jean de Stilo (1336), Thomas de Stilo (1343), Marin (1346), Patermanus d'Ancône (1349) et Michel de Lecce (1355, chirurgien communal encore à la date de son décès en 1371). Leurs salaires annuels varient de 4 à 10 livres. Jean de Stilo, originaire de Salerne, recevait 15 livres, car il était à la fois chirurgien et pharmacien de la ville. Un autre Salernitain, de la même famille, rendait ce double service pour 12 livres $^{25}$.

Pendant la même période, de 1319 à 1358, la commune de Raguse payait les médecins-physiciens suivants: Guillaume (1320), Uguiccio de Padoue 
(1322), Giramont (1322), Jean de Anterminellis de Lucques (1323), Gilles (1324), Jean d'Aquilée (1324), Antoine de Montefiore (1329), Nicolas de Padoue (1343), Jacques de Padoue (1344), Benoît (1354) et Gérard de Ravenne $(1354)^{26}$.

Pendant au moins trois ans, la ville eut à son service un médecin français, maître Giramont (ou Garamond). Les documents officiels lui donnent le titre de medicinae professor ${ }^{27}$. Ce titre est attribué aussi à deux autres physiciens salariés, maître Uguiccio de Padoue et maître Jean de Anterminellis de Lucques. Les salaires annuels de ces trois médecins, exerçant à Raguse en même temps, varient entre 12 et 20 livres, l'indemnité pour le logement non comprise. Comme les chirurgiens salariés, ils devaient soigner gratuitement tous les citoyens de Raguse, mais sans obligation de fournir les médicaments. Il était précisé qu'ils ne pouvaient recevoir en cadeau que des comestibilia parvi valoris et quantitatis, c'est-à-dire des vivres de valeur symbolique (tel ce coq traditionnel que, sur un bas-relief du $\mathrm{XVI}^{e}$ siècle, sculpté sur le Palais ducal de Raguse, un patient apporté au médecin).

En 1324, le Grand Conseil autorise le Duc et le Petit Conseil à prendre au service de la commune un médecin juif. Sans doute, c'était la religion de maître Gilles, physicien salarié de 1324 à 1329 , avec un traitement de 11 livres. A deux reprises, la République de Raguse a mis ce médecin à la disposition du roi serbe Etienne III. Pour le maître Benoît, on n'oublie pas de mentionner dans les actes officiels qu'il est d'origine juive (olim Judeus). En 1357, la commune lui a accordé la permission de se rendre à Jérusalem, sur la tombe du Christ, mais avec la suspension du salaire pendant son absence de Raguse ${ }^{28}$.

En 1331, le Grand Conseil décide qu'«aucun médecin salarié, aussi bien physicien que chirurgien, n'est autorisé à exercer un autre métier ou commerce à Raguse et, sous peine de perdre son salaire, ne peut entrer en association avec un pharmacien, ni avoir une part dans sa boutique ${ }^{29}$.

\section{De l'indépendance à la fin du $\mathrm{XV}^{\mathrm{e}}$ siècle}

Depuis son accession à l'indépendance au milieu du XIV siècle jusqu'à la fin du Moyen Age, la République de Raguse continue d'assurer à ses sujets un service médical de haute qualité.

Les actes officiels mentionnent les chirurgiens salariés suivantes: Cobel-

26 J.-T., ibid.

27 Mon. Rag. I 59 et 116. Vor J.-T., II 10 et III 129-132, et M. Deanović, Anciens contacts entre le France et Raguse, Zagreb, 1950, p. 24-25.

28 Mon. Rag. II 149 et 196.

29 AED, Diversa Cancellariae 10,18. 
lus (1360), Thaddée de Ferrare (1360), Thomas de Nomais de Forli (1363), Barthélemy (1366), Jean de Trogir (1375), Jean de Aldoardis de Pavie (1376), Pierre de Venise (1382), Grégoire de Vérone (1383), Denis (1387), André de Pellagiis de Bari (1392), Urbain (1403), Pierre de Bologne (1403), Jean d'Ancone (1403), Jean de Recanati (1412), Jean de Teolo de Padoue (1423), Jean Matthieu de Reginis de Feltre (1436), Pierre fils de Jean de Reginis (1452), Barthélemy (1455), Jean de la Dolce Calderia de Naples (1455), Jean de Crète (1461), Jean d'Espagne (1482), Michel de Kotor (1483) et Jacques Catalan de Barcelone (1486) $)^{30}$.

La liste des physiciens salariés n'est pas moins bien fournie que celle des chirurgiens: Christophe de Bénévent (1360), Guillaume de Ravenne (1361), François de Fermo (1366), Pierre de Ricombaldis de Bari (1381), Albertin de Camurata de Padoue (1385), Balthazar de Padoue (1399), Jacques de Salgheriis de Padoue (1402), Pierre fils d'Albertin de Camurata de Venise (1407), Daniel de Pasinis de Vérone (1407), Barthélemy de Squarcialupi de Piombino (1409), Thomas fils du maître Jean de Pavie (1409), Jacques Gondoaldo de Ferrare (1416), Jacques Mazia de Salerne (1426), Jean Coronelli de Conegliano (1427), Christophor de Bonasis de Padoue (1436), Jacques de Protonothariis de Messine (1436), Georges Spanus (1444), Jean Evangeliste de Caversalis de Imola (1444), Ange Conti de Venise (1449), André de Constantinople (1458), Jean François de Trévise (1460), Thaddée Ardoino de Florence (1461), Jean Pierre de la Varugola de Vérone (1461), Emmanuel Marulla de Corfù (1465), Jean Clado (1466), Jérôme de Udine (1467), Hector Pindemonte de Vérone (1471), Gabriel de Galvano (1476), Jérôme Conti de Urbino (1482), Antoine Vittori de Faenza (1490) et François de Valencia (1497) ${ }^{31}$.

Une personnalité hors pair était le maître Christophe de Bénévent, entré au service de Raguse en 1360. Il était physicien salarié au moment où cette ville portuaire a institué, la première au monde, la quarantaine comme mesure de protection contre la peste (1377). En octobre 1399, le Grand Conseil décide de ne plus prolonger son contrat mais, vu les services rendus, de lui assurer, aux frais de la commune et jusqu'à la fin de ses jours, une pension de 10 perpères. Peu de temps après, le gouvernement se voit obligé de nommer trois curateurs - un prêtre, un pharmacien et le fils de l'intéressé - pour prendre soin des affaires du maître Christophe qui «à cause de l'âge a perdu sa raison». Le vieux médecin n'a survécu que quelques mois dans cet état de déchéance ${ }^{32}$.

30 J.-T., II 13-45 et III 132-144.

31 J.-T., ibid.

32 Voir J. Bačić, «Magister Kristofor fizik (prva liječnička mirovina u starom Dubrovniku)», Liječnički Vjesnik, 108, 1986, 108-110; M. D. Grmek, «Le concept d'infection dans l'Antiquité 
Trois autres médecins méritent une mention particulière. Barthélemy de Squarcialupi de Piombino, médecin-physicien salarié à Raguse de 1409 à 1426, a écrit un ouvrage sur la peste, dédié à Sigismond, roi de Hongrie et de Croatie $^{33}$. Arrivé à Raguse en 1416, Jacques Gondoaldo de Ferrare, artium et medicinae doctor, a été d'abord employé comme rector scolarum, puis comme physicien salarié (de 1430 à sa mort en 1437). Il a obtenu des succès remarquables dans la protection épidémiologique de la ville, notamment par l'amélioration des conditions de quarantaine et d'isolement des malades ${ }^{34}$. Jacques Mazia de Salerne, à la fois physicien et chirurgien, a promis par un contrat passé devant notaire le 25 juin 1426 de guérir Bogdan Bogcinovich d'un ulcère à la jambe et cela en opérant la grande veine «dont cette maladie provient et dépend $»^{35}$.

Pendant la seconde moitié du XIV et tout au long du XV siècle, les remunérations des médecins communaux étaient fixées, par décision du conseil municipal ou par contrats, en ducats d'or ou en perpères et non plus en livres vénitiennes. Les salaires variaient considérablement, selon la rénommée du médecin et encore plus selon les nécessités sanitaires et selon les circonstances politiques. Les physiciens recevaient entre 200 et 400 ducats, quelques-uns seulement 150 ducats et un docteur de Padoue exceptionnellement 500 ducats par an. Les chirurgiens n'obtenaient qu'environ 150 ducats.

\section{Conclusions}

Les physiciens salariés étaient toujours des étrangers ayant reçu leur formation professionnelle dans les villes universitaires italiennes, notamment à $\mathrm{Pa}$ doue, Salerne, Bologne et Ferrare ${ }^{36}$. Les Italiens étaient de loin les plus nombreux, mais la commune a engagé aussi quelques Espagnols et Grecs, un Français et un Allemand. Plusieurs médecins espagnols et italiens d'origine juive ont librement exercé leur art sur le territoire de la petite République. Aucun des Ragusains qui ont obtenus le doctorat en médecine avant 1500 n'est revenu dans sa ville natale.

et au Moyen Age, les anciennes mesures sociales contre les maladies contagieuses et la fondation de la première quarantaine à Dubrovnik», Rad. J. A. (Zagreb), 384, 1980, 9-55, et J.T., II 13-14.

33 Voir E. Schultheiss, «Colcodei seu liber de peste des Bartholomeus Squarcialupi de Plumbino», Sudhoffs Archiv für Geschichte der Medizin, 44, 1960, 333-340.

34 Voir R. Bernabeo, «Del ferrarese maestro Giacomo Gondoaldo medico fisico salariato della Repubblica di Ragusa», L'Arcispedale S. Anna di Ferrara, 22, 1969, 1001-1034.

35 AED, Diversa Notariae 15, 3. - M. D. Grmek, «Influence de l'Ecole de Salerne sur le développement de la médecine en Croatie», Scalpel, 106, 1953,33-37.

36 Voir M. D. Grmek, «O stručnoj naobrazbi liječnika u Hrvatskoj do početka XVIII stoljeća», Liječnički Vjesnik, 90, 1968, 1221-1238. 
Les chirurgiens salariés étaient le plus souvent des étrangers, mais pas nécessairement. Il étaient formés par l'apprentissage, la majorité en Italie, notamment à Venise, quelques-uns en Dalmatie.

Les devoirs des physiciens salariés étaient de visiter à leur domicile les citoyens qui demandaient leur secours (jusqu'à deux fois par jour, précisent certains contrats), de soigner les maladies internes en prescrivant le traitement, de surveiller le travail des chirurgiens, des pharmaciens et des sagesfemmes et, enfin, de signaler l'apparition des maladies pestilentielles et de donner au gouvernement des conseils concernant la santé publique. Il leur était interdit de participer au commerce des médicaments et d'avoir des accords particuliers avec des pharmaciens. Sans une autorisation spéciale, ils ne pouvaient s'absenter de la ville. Ils fournissaient des certificats médicaux, permettant par exemple d'être dispensé d'une charge administrative ou d'une mission politique.

Les chirurgiens soignaient les plaies, s'occupaient des fractures, ouvraient des abcès, cautérisaient, pratiquaient la saignée et, d'une manière générale, exécutaient les ordres des physiciens concernant les modalités du traitement. Ils devaient dénoncer des blessures dues aux rixes ou aux actes criminels et agissaient souvent comme experts auprès des tribunaux.

Les contrats d'engagement prévoyaient le plus souvent un ou deux ans de service avec des prolongements d'année en année. Un bon nombre de ces médecins sont resté à Raguse dix ans ou plus. Plusieurs sont décédés dans l'exercice de leurs fonctions. Les testaments conservés dans les archives notariales donnent un aperçu instructif sur les instruments dont ils se servaient et sur les livres qu'ils lisaient. Ainsi, par exemple, les inventaires des successions d'Antoine de Montefiore (1337), de Pierre de Camurata (1418), de Jacques de Protonothariis (1442) et de Jean Matthieu de Reginis (1454) révèlent la diversité des intérêts scientifiques et culturels de ces médecins et illustrent leur rôle dans la diffusion des idéaux humanistes ${ }^{37}$.

37 AED, Testamenta 3,57; Diversa Notariae 12,256; Diversa Cancellariae 56, 115; Testamenta 15 , 90. 